\title{
Acoustoelectric Effect in Fluorinated Carbon Nanotube in the Absence of External Electric Field
}

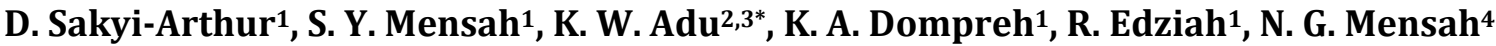 \\ ${ }^{1}$ Department of Physics, College of Agriculture and Natural Sciences, U.C.C, Cape Coast, Ghana \\ ${ }^{2}$ Department of Physics, Pennsylvania State University-Altoona College, Altoona, PA, USA \\ ${ }^{3}$ Material Research Institute, Pennsylvania State University, University Park, PA, USA \\ ${ }^{4}$ Department of Mathematics, College of Agriculture and Natural Sciences, U.C.C, Cape Coast, Ghana \\ Email: *cxa269@psu.edu
}

How to cite this paper: Sakyi-Arthur, D., Mensah, S.Y., Adu, K.W., Dompreh, K.A., Edziah, R. and Mensah, N.G. (2020) Acoustoelectric Effect in Fluorinated Carbon Nanotube in the Absence of External Electric Field. World Journal of Condensed Matter Physics, 10, 1-11.

https://doi.org/10.4236/wjcmp.2020.101001

Received: December 17, 2019

Accepted: January 28, 2020

Published: January 31, 2020

Copyright $\odot 2020$ by author(s) and Scientific Research Publishing Inc. This work is licensed under the Creative Commons Attribution-NonCommercial International License (CC BY-NC 4.0). http://creativecommons.org/licenses/by-nc/4.0/ (c) (i) (8) Open Access

\begin{abstract}
Acoustoelectric effect (AE) in a non-degenerate Fluorine modified single walled carbon nanotube (FSWCNT) semiconductor is studied theoretically using the Boltzmann's transport equation. The study is done in the hypersound regime i.e. $q \ell \gg 1$, where $q$ is the acoustic phonon wavenumber and $\ell$ is the electron mean free path. The results obtained are compared with that of undoped single walled carbon nanotube (SWCNT). The AE current density for FSWCNT is observed to be four orders of magnitude smaller than that of undoped SWCNT with increasing temperature, that is $\left|j_{z}^{F S W C N T}\right| \ll\left|j_{z}^{S W C N T}\right|$. This is because the electron-phonon interactions in SWCNT are stronger than FSWCNT. Thus, there are more intra-mini-band electrons interacting with the acoustic phonons to generate a higher AE current in SWCNT than in FSWCNT. This has been observed experimentally, where the electrical resistance of FSWCNT is higher than pristine SWCNT i.e. $R \gg 20 \mathrm{M} \Omega$. The study shows the potential for FSWCNT as an ultrasound current source density imaging (UCSDI) and AE hydrophone material. However, FSWCNT offers the potential for room temperature applications of acoustoelectric device but other techniques are needed to reduce the resistance.
\end{abstract}

\section{Keywords}

Carbon Nanotube, Fluorinated, Acoustoelectric, Hypersound

\section{Introduction}

The absorption of acoustic waves in semiconductors leads to the transfer of 
energy and momentum from the acoustic phonons to the charge carriers. This exchange sets up a $d c$ current called acoustoelectric (AE) current density $\left(j^{A E}\right)$ [1] [2] [3] [4] and thus give rise to AE current in the case of an open circuit, or a constant electric field $\boldsymbol{E}(t)$ [5] [6]. Acoustoelectricity has applications in biomedical engineering [7], metrology [8] and quantum information processing [9] [10]. The confinement of electrons in one and two dimensional structures, modifies the electron mobility remarkably, thus reducing electron-phonon interaction, and scattering rate [11] [12] [13] to enable electrons to move with ease. This further increases the $d c$ electrical conductivity, due to size effect [14] [15] [16]. Research into AE is very relevant, due to the complementary role it plays in understanding the thermal, electrical and electronic properties of semiconductors and its position in acoustoelectronics.

$\mathrm{AE}$ has been studied extensively over the past decade in semiconductor superlattices (SLs) [6] [7], graphene [17] and recently in single-walled carbon nanoutbes (SWCNT) [18] [19]. Other studies such as high electron thermal conductivity in SWCNT [20], hot-electron effect in quasi-static field in SWCNT [21] and absorption coefficient of acoustic phonons in FSWCNT [22] have been carried out.

Doping a metallic SWCNT with Fluorine and thus ensuring a double periodicity along the axial direction forms a FSWCNT as perceived by Sadykov et al. [23], thus, changing the metallic property to semiconducting. The generation of AE current for contemporary applied sciences as against the use of conventional direct current has attracted lots of interest in heterogenous structured devices [24]. Studies done in SLs, homogenous semiconductors, graphene and SWCNT have proven that carbon nanotube is a good candidate for such studies due to their outstanding physical properties that stem from high scattering mechanisms, high bias mean free path and their reduced dimensions which permit strong electron-phonon interactions to occur, thus, resulting in strong acoustic phonon scattering [11].

Electron confinement along their circumference makes them behave as quasi one-dimensional structure characterized by delocalized $\pi$-electrons in $s p^{2}$-hybridized state. Experimental studies conducted by Jeon et al. [25] have shown that absorption of acoustic waves in FSWCNT is far less than that of undoped SWCNT and studies on the absorption coefficient of acoustic phonons in FSWCNT in the hypersound regime in the absence of an external electric field was done recently to confirm this theoretically [22]. Even though there has been report on the optical properties of acoustic phonon effect in FSWCNT [22], up to date no study on electronic properties due to $\mathrm{AE}$ in FSWCNT in the hypersound regime has been carried out. In this paper, we perform a theoretical investigation of the effect of stimulating FSWCNT with acoustic phonon without an electric field in the hypersound regime and its potential as an ultrasound current source density imaging (UCSDI) and AE hydrophone material. 


\section{Theory}

For a chemically modified SWCNT with Fluorine, where the Fluorine atoms form a one-dimensional chain, the energy dispersion can be deduced by using the Huckel matrix method as in Equation (1). Accounting for translational symmetry [23]

$$
\varepsilon\left(p_{z}\right)=\varepsilon_{o}+\Xi_{n} \gamma_{o} \cos ^{2 N-1}\left(a p_{z}\right)
$$

where $a=\sqrt{3} b / 2 \hbar, \Xi$ is a constant, $\gamma_{o}$ is the overlapping integral, $p_{z}$ is the dynamic momentum of an electron along the axial direction (z-axis), $N$ is an integer and $\varepsilon_{o}$ is the electron energy in the first Brillouin zone with momentum $p_{o}$, i.e. $-\pi / a \leq p_{o} \leq \pi / a$. For $N=2$, the energy dispersion for FSWCNT at the Fermi surface at the edge of the Brillouin zone is

$$
\varepsilon\left(p_{z}\right)=\varepsilon_{o}+8 \gamma_{0} \cos ^{3}\left(a p_{z}\right)
$$

Equation (2) can be expanded as

$$
\varepsilon\left(p_{z}\right)=\varepsilon_{o}+\Delta_{1} \cos \left(3 a p_{z}\right)+\Delta_{2} \cos \left(a p_{z}\right)
$$

where $\Delta_{1}=2 \gamma_{o}$ and $\Delta_{2}=6 \gamma_{o}$.

Consider a single travelling longitudinal acoustic wave, moving along a uniform tube of FSWCNT with the ends of the tube being electrically insulated. The travelling acoustic wave is induced by driving one end of the tube with a vibrator while matching the other end to a proper acoustic impedance to ensure no reflection of the wave at the termination. The ends of the tube being electrically insulated, the acoustic wave would drag conduction electrons to one end of the tube, creating a deficiency of electrons at the other end. The resultant electric field along the tube will generate a conventional electric current which exactly cancels the current associated with the acoustoelectric effect. The acoustoelectric effect, therefore, may be measured by determining the electric potential difference between the tyro ends of the tube. Strikingly, this process is analogous to the voltage generation in a open circuit in thermoelectric effect due to temperature difference. The net flow of phonons along a temperature gradient may be considered as a net flow of travelling acoustic waves along the gradient.

Assume the sound wave and the constant electric field travel along the $\mathrm{z}$-axis of the FSWCNT, then the AE current density in the FSWCNT is defined as [6] [18]:

$$
j^{A E}=-e \sum_{n, n^{\prime}} \int U_{n, n^{\prime}}^{a c} \Psi_{i}\left(p_{z}\right) \mathrm{d}^{2} p_{z}
$$

where $\Psi_{i}\left(\boldsymbol{p}_{z}\right)$ is the solution to the Boltzmann kinetic equation in the absence of a magnetic field, and the kinetic equation is given as:

$$
\boldsymbol{v} \frac{\partial \Psi_{i}}{\partial \boldsymbol{p}}+\boldsymbol{W}_{p}\{\Psi\}=v_{i}
$$

$\boldsymbol{p}_{z}$ is the electron momentum along the axial direction of the FSWCNT and $U_{n, n^{\prime}}^{a c}$ in Equation (4) is the electron-phonon transition defined in refs. [18] [22] [26] as: 


$$
\begin{aligned}
U_{n, n^{\prime}}^{a c}= & \frac{2 \pi \Phi}{\omega_{q} v_{s}} \sum_{n, n^{\prime}}\left\{\left|G_{p_{z}-\hbar q, p_{z}}\right|^{2}\left[f\left(\varepsilon_{n}\left(p_{z}-\hbar q\right)\right)-f\left(\varepsilon_{n}\left(p_{z}\right)\right)\right]\right. \\
& \times \delta\left(\varepsilon_{n}\left(p_{z}-\hbar q\right)-\varepsilon_{n}\left(p_{z}\right)+\hbar \omega_{q}\right) \\
& +\left|G_{p_{z}+\hbar q, p_{z}}\right|^{2}\left[f\left(\varepsilon_{n^{\prime}}\left(p_{z}+\hbar q\right)\right)-f\left(\varepsilon_{n^{\prime}}\left(p_{z}\right)\right)\right] \\
& \left.\times \delta\left(\varepsilon_{n^{\prime}}\left(p_{z}+\hbar q\right)-\varepsilon_{n^{\prime}}\left(p_{z}\right)-\hbar \omega_{q}\right)\right\}
\end{aligned}
$$

where $f\left(p_{z}\right)=f\left(\varepsilon_{n, n^{\prime}}\left(p_{z}\right)\right)$ is the electron distribution function, $\Phi$ is the sound flux density, $\varepsilon_{n, n^{\prime}}\left(p_{z}\right)$ is the electron energy band, and $n$ and $n^{\prime}$ denote the quantization of the energy band induced by the wavevector as a result of the periodic boundary conditions imposed on the wavefunction in the circumferential direction. $G\left(p_{z} \pm \hbar q, p_{z}\right)$ is the matrix element of the electron-phonon interaction. Employing the principle of detailed balance and denoting $p_{z}^{\prime}=p_{z} \pm \hbar q$, we obtain the condition:

$$
\left|G_{p^{\prime}, p}\right|^{2}=\left|G_{p, p^{\prime}}\right|^{2}
$$

and the matrix element of the electron-phonon interaction is given as:

$$
\left|G_{p^{\prime}, p}\right|=\frac{4 \pi e K}{\sqrt{2 \rho \omega_{q}} \varepsilon}
$$

where $K$ is the piezoelectric modulus, $\varepsilon$ is the lattice dielectric constant, $\rho$ is the density of FSWCNT. The AE current density then takes the form

$$
\begin{aligned}
j_{z}^{A E}= & -\frac{2 e}{(2 \pi \hbar)^{2}} \frac{\pi \Phi}{\omega_{q} v_{s}} \sum_{n, n^{\prime}}\left|G_{p_{z}^{\prime}, p_{z}}\right|^{2}\left[f\left(\varepsilon_{n^{\prime}}\left(p_{z}\right)\right)-f\left(\varepsilon_{n^{\prime}}\left(p_{z}+\hbar q\right)\right)\right] \\
& \times\left[\Psi_{i}\left(p_{z}+\hbar q\right)-\Psi_{i}\left(p_{z}\right)\right] \delta\left(\varepsilon_{n^{\prime}}\left(p_{z}+\hbar q\right)-\varepsilon_{n^{\prime}}\left(p_{z}\right)-\hbar \omega_{q}\right) \mathrm{d} p_{z}
\end{aligned}
$$

where, $\Psi_{i}\left(p_{z}\right)=l_{i}\left(p_{z}\right)$ [26] is the electron mean free path written as:

$$
l_{z}=\tau v_{z}
$$

and the electron group velocity is expressed as:

$$
v_{z}=\frac{\partial \varepsilon\left(p_{z}\right)}{\partial p_{z}}
$$

Substituting Equation (10) and Equation (11) into Equation (9) yields:

$$
\begin{aligned}
j_{z}^{A E}= & -\frac{16 e^{3} \pi \Phi K^{2} \tau}{2 \varepsilon^{2} \omega_{q}^{2} v_{s} \rho \hbar^{2}} \sum_{n, n^{\prime}}\left[f\left(\varepsilon_{n^{\prime}}\left(p_{z}\right)\right)-f\left(\varepsilon_{n^{\prime}}\left(p_{z}+\hbar q\right)\right)\right] \\
& \times\left[v_{z}\left(p_{z}+\hbar q\right)-v_{z}\left(p_{z}\right)\right] \delta\left(\varepsilon_{n^{\prime}}\left(p_{z}+\hbar q\right)-\varepsilon_{n^{\prime}}\left(p_{z}\right)-\hbar \omega_{q}\right) \mathrm{d} p_{z}
\end{aligned}
$$

The electron distribution function is given by the shifted Fermi-Dirac distribution as

$$
f_{o}(p)=\frac{1}{1+\exp \left[\left(\varepsilon\left(p_{z}\right)-\mu\right) / k T\right]}
$$

where $k$ is Boltzmann's constant, $T$ is the absolute temperature in energy units, and $\mu$ is the electrochemical potential. Substituting Equation (13) into Equation (12) we obtain an equation with the term $\mathcal{F}_{1 / 2}$, representing Fermi-Dirac 
integral of the order $1 / 2$, and written as:

$$
\begin{aligned}
j_{z}^{A E}= & -\frac{16 e^{3} \pi \Phi K^{2} \tau}{2 \varepsilon^{2} \omega_{q}^{2} v_{s} \rho \hbar^{2}} \sum_{n, n^{\prime}}\left[\mathcal{F}_{1 / 2}\left(\varepsilon_{n^{\prime}}\left(p_{z}\right)\right)-\mathcal{F}_{1 / 2}\left(\varepsilon_{n^{\prime}}\left(p_{z}+\hbar q\right)\right)\right] \\
& \times\left[v_{z}\left(p_{z}+\hbar q\right)-v_{z}\left(p_{z}\right)\right] \delta\left(\varepsilon_{n^{\prime}}\left(p_{z}+\hbar q\right)-\varepsilon_{n^{\prime}}\left(p_{z}\right)-\hbar \omega_{q}\right) \mathrm{d} p_{z}
\end{aligned}
$$

The Fermi-Dirac integral $\mathcal{F}_{1 / 2}$, is given as:

$$
\mathcal{F}_{1 / 2}\left(\eta_{f}\right)=\frac{1}{\Gamma(1 / 2)} \int_{0}^{\infty} \frac{\eta_{f}^{1 / 2} \mathrm{~d} \eta}{1+\exp \left(\eta-\eta_{f}\right)}
$$

where $\left(\mu-\varepsilon_{c}\right) / k T \equiv \eta_{f}$ and $\Gamma(1 / 2)$ is the Gamma function of the order $1 / 2$. For nondegenerate electron gas, where the Fermi level is several $k T$ below the energy of the conduction band edge $\varepsilon_{c}$, (i.e. $k T \ll \varepsilon_{c}$ ), the integral in Equation (15) approaches $\sqrt{\pi} / 2 \exp \left(\eta_{f}\right)$ and so Equation (12) becomes:

$$
f_{o}(p)=A^{\dagger} \exp \left(-\frac{\varepsilon(p)-\mu}{k T}\right)
$$

The electron distribution function in the presence of the applied constant electric field, $E(t)$ is obtained by solving the Boltzmann equation in the $\tau$-approximation as:

$$
f\left(p_{z}\right)=\int_{0}^{\infty} \frac{\mathrm{d} t}{\tau} \exp (-t / \tau) f_{o}\left(p_{z}-p^{\prime}\right)
$$

and $A^{\dagger}$ is the normalization constant to be determined from the normalization condition $\int f(\boldsymbol{p}) \mathrm{d} \boldsymbol{p}=n_{o}$ as:

$$
A^{\dagger}=\frac{3 n_{o} a^{2}}{2 I_{o}\left(\Delta_{1}^{*}\right) I_{o}\left(\Delta_{2}^{*}\right)} \exp \left(\frac{\varepsilon_{o}-\mu}{k T}\right)
$$

where $n_{o}$ is the electron concentration and $I_{o}(x)$ is the modified Bessel function of zero order and all other parameters are as defined previously.

Assuming the electrons are confined to the lowest conduction band, then $n=n^{\prime}=1$. The electron velocity obtained from the dispersion relation is given as

$$
v_{z}\left(\boldsymbol{p}_{z}\right)=-\left[3 a \Delta_{1} \sin \left(3 a \boldsymbol{p}_{z}\right)+a \Delta_{2} \sin \left(a \boldsymbol{p}_{z}\right)\right]
$$

Substituting Equation (16)-Equation (19) into Equation (14), the AE current density is obtained as:

$$
\begin{aligned}
j_{z}^{A E}= & \frac{4 A^{\dagger} \pi \Phi e^{3} K^{2} \tau \Theta\left(1-\alpha^{2}\right)}{\hbar^{3} \omega_{q}^{2} \varepsilon^{2} v_{s} \rho a q \sqrt{1-\alpha^{2}}} \int_{0}^{\infty} \exp \left(\frac{\mathrm{d} t^{\prime}}{\tau}\right) \\
& \times\left\{\sinh \left[\Delta_{1}^{*} \cos \left(3 e a \boldsymbol{E} t^{\prime}\right) \sin A \sin \left(\frac{3}{2} a \hbar q\right)+\Delta_{2}^{*} \cos \left(e a \boldsymbol{E} t^{\prime}\right) \sin B \sin \left(\frac{a}{2} \hbar q\right)\right]\right. \\
& \times \sinh \left[\Delta_{1}^{*} \cos \left(3 e a E t^{\prime}\right) \cos A \cos \left(\frac{3}{2} a \hbar q\right)+\Delta_{2}^{*} \cos \left(e a \boldsymbol{E} t^{\prime}\right) \cos B \cos \left(\frac{a}{2} \hbar q\right)\right] \\
& -4\left(\Delta_{2}^{*} \sin \left(e a \boldsymbol{E} t^{\prime}\right) \cos B \sin \left(\frac{a}{2} \hbar q\right)+\Delta_{1}^{*} \cos A \sin \left(3 e a E t^{\prime}\right) \sin \left(\frac{3}{2} a \hbar q\right)\right.
\end{aligned}
$$




$$
\begin{aligned}
& \left.+\Delta_{1}^{*} \Delta_{2}^{*} \sin \left(e a E t^{\prime}\right) \sin \left(3 e a E t^{\prime}\right) \cos A \cos B \sin \left(\frac{a}{2} \hbar q\right) \sin \left(\frac{3}{2} a \hbar q\right)\right) \\
& \times \cosh \left[\Delta_{1}^{*} \cos \left(3 e a E t^{\prime}\right) \cos A \cos \left(\frac{3}{2} a \hbar q\right)+\Delta_{2}^{*} \cos \left(e a E t^{\prime}\right) \cos B \cos \left(\frac{a}{2} \hbar q\right)\right] \\
& \left.\times \cosh \left[\Delta_{1}^{*} \cos \left(3 e a E t^{\prime}\right) \sin A \sin \left(\frac{3}{2} a \hbar q\right)+\Delta_{2}^{*} \cos \left(e a E t^{\prime}\right) \sin B \sin \left(\frac{a}{2} \hbar q\right)\right]\right\}
\end{aligned}
$$

where $\Delta_{1}^{*}=\Delta_{1} / k T$, and $\Delta_{2}^{*}=\Delta_{2} / k T$. Switching off the external electric field $(\boldsymbol{E}=0)$, Equation (20) reduces to:

$$
\begin{aligned}
j_{z}^{F S W C N T}= & \frac{4 A^{\dagger} \pi \Phi e^{3} K^{2} \tau \Theta\left(1-\alpha^{2}\right)}{\hbar^{3} \omega_{q}^{2} \varepsilon^{2} v_{s} \rho a q \sqrt{1-\alpha^{2}}} \\
& \times\left[\sinh \left\{\Delta_{1}^{*} \sin \left(\frac{3}{2} a \hbar q\right) \sin A+\Delta_{2}^{*} \sin \left(\frac{a}{2} \hbar q\right) \sin B\right\}\right. \\
& \left.\times \sinh \left\{\Delta_{1}^{*} \cos \left(\frac{3}{2} a \hbar q\right) \cos A+\Delta_{2}^{*} \cos \left(\frac{a}{2} \hbar q\right) \cos B\right\}\right]
\end{aligned}
$$

Simplifying Equation (21) yields:

$$
\begin{aligned}
j_{z}^{\text {FSWCNT }}= & j_{o}\left[\sinh \left\{\Delta_{1}^{*} \sin \left(\frac{3}{2} a \hbar q\right) \sin A+\Delta_{2}^{*} \sin \left(\frac{a}{2} \hbar q\right) \sin B\right\}\right. \\
& \left.\times \sinh \left\{\Delta_{1}^{*} \cos \left(\frac{3}{2} a \hbar q\right) \cos A+\Delta_{2}^{*} \cos \left(\frac{a}{2} \hbar q\right) \cos B\right\}\right]
\end{aligned}
$$

where

$$
j_{o}=\frac{6 A^{\dagger} \pi \Phi e^{3} K^{2} a \tau \Theta\left(1-\alpha^{2}\right)}{\hbar^{3} \omega_{q}^{2} \varepsilon v_{s} \rho q \sqrt{1-\alpha^{2}}}, \alpha=\frac{\omega_{q}}{12 \gamma_{o} a q}
$$

and

$$
A=\frac{3}{4} \sin ^{-1}\left(\frac{\omega_{q}}{12 \gamma_{o} a q}\right), B=\frac{1}{4} \sin ^{-1}\left(\frac{\omega_{q}}{12 \gamma_{o} a q}\right)
$$

and $\Theta$ is a Heaviside step function.

To compare the results obtained with that of undoped SWCNT, we follow the same procedure for the FSWCNT. Using the tight-binding energy dispersion of the $p_{z}$ orbital which is given as:

$$
\varepsilon\left(p_{z}\right)= \pm \gamma_{o}\left(1-2 \cos \left(\frac{p_{z} \sqrt{3} b}{2 \hbar}\right)\right)
$$

From Equation (23), the acoustocurrent density in undoped SWCNT obtained via deformation potential is expressed as:

$$
j_{z}^{S W C N T}=j_{o}^{S W C N T} \sinh \left\{\frac{\hbar \omega_{q}}{k T}\right\} \sinh \left\{\Delta^{*} \sqrt{1-\alpha^{2}} \cos \left(\frac{a \hbar q}{2}\right)\right\}
$$

where 


$$
j_{o}^{S W C N T}=\frac{\Lambda^{2} q^{2} e \tau n_{o} a \Theta\left(1-\alpha_{o}^{2}\right)}{\pi \hbar^{2} \omega_{q}^{2} v_{s} \rho \sin (a \hbar q / 2) I_{o}\left(\Delta^{*}\right) \sqrt{1-\alpha_{o}^{2}}}
$$

and

$$
\alpha_{o}=\frac{\hbar \omega_{q}}{4 \gamma_{o} \sin (a \hbar q / 2)}, \Delta=4 \gamma_{o}, \Delta^{*}=\Delta / k T
$$

\section{Results and Discussion}

The acoustic wave considered has a wavelength $\lambda=2 \pi / q$, smaller than the mean-free path of FSWCNT electrons in the hypersound region $q \ell \gg 1$. The acoustic wave is then treated as packets of coherent phonons (monochromatic phonons) having a $\delta$-function distribution as

$$
N(\boldsymbol{k})=\frac{(2 \pi)^{3}}{\hbar \omega_{q} v_{s}} \boldsymbol{\Phi} \delta(\boldsymbol{k}-\boldsymbol{q})
$$

where $\boldsymbol{k}$ is the electron wavevector, $\hbar$ is the reduced Planck's constant, $\boldsymbol{\Phi}$ is the sound flux density, and $\omega_{q}$ and $v_{s}$ are respectively the frequency and the group velocity of sound wave with wavevector $\boldsymbol{q}$. The general equations for the acoustoelectric current density in FSWCNT $\left(j_{z}^{\text {FWCNT }}\right)$ and SWCNT $\left(j_{z}^{S W C N T}\right)$ are presented in Equation (22) and Equation (24), respectively. The acoustocurrent generated in both cases is observed to be strongly dependent on acoustic wavenumber $(q)$, frequency $\left(\omega_{q}\right)$ and the temperature $(T)$. A transparency window is observed when $\omega_{q} \gg 12 \gamma_{o} a q$ and $\omega_{q} \gg 4 \gamma_{o} \sin (a \hbar q / 2) / \hbar$ for FSWCNT and SWCNT respectively, consequence of the conservation laws. It follows then that, only electrons with momenta $\hbar q / 2$ interacts with the phonons. If the sound flux passing through the sample has a frequency which is extremely high, there will be no absorption of sound and the acoustoelectric current will be zero.

From Equation (22) and Equation (24), when $j_{z}^{A E}=0$, the dependence on $q$ is highly nonlinear as observed in [13] [26] for the condition that

$$
G\left(p_{z}, \hbar q\right)=i b^{*}\left[\sin \left(p_{z}+\hbar q\right) d-\sin p_{z} d\right]
$$

where $b^{*}$ is the derivative of the resonance integral with respect to the interatomic distance. Equation (22) and Equation (24) are analyzed numerically with the following parameters: $\omega_{q}=10^{12} \mathrm{~s}^{-1}, \quad v_{\mathrm{s}}=2.5 \times 10^{3} \mathrm{~m} / \mathrm{s}, \quad \Phi=10^{5} \mathrm{~Wb} / \mathrm{m}^{2}$ and $\ell=10^{-4} \mathrm{~cm}$.

The AE current density is obtained under quasi-static field $\omega \tau \gg 1$ by solving the Boltzmann's transport equation with constant relaxation time $\tau$. The acoustocurrent density exhibits linear dependence on $q$ at $E=0$ (in the region of ohmic conductivity). As $q$ increases the acoustoelectric current density increases to a maximum, and drops off, experiencing a negative conductivity as shown in Figure 1. The acoustoelectric current density obtained in Figure 1(a) for FSWCNT is four orders of magnitude lesser than that obtained in Figure 1(b) for SWCNT. This is because the electron-phonon interactions in SWCNT are 
stronger than FSWCNT. Thus there are more intra-mini-band electrons interacting with the acoustic phonons to generate the acoustoelectric current in SWCNT than in FSWCNT. Furthermore, more phonons of high wavevectors $q \approx 10^{9} \mathrm{~cm}^{-1}$ participate in the energy/momentum transfer interactions in SWCNT than in FSWCNT $q \approx 10^{6} \mathrm{~cm}^{-1}$. The nonlinear rise and fall is attributed to the Bloch oscillations of the intra-mini-band electron current which is a consequence of Bragg's reflections at the band edges [27]. Since the electrons are under a lattice potential field, the phonons trade their energy and momentum to the electrons which move within the Brillouin zone. When they reach the top of the Brillouin zone, they acquire certain momenta and are reflected from the edges of the Brillouin zone (Bragg's reflection). It is clear from Figure 1 that increasing the temperature decreases the acoustoelectric current density and shifts the peak to higher $q$ (wavevectors) and this is due to Bragg's reflection and the nonparabolicity of the dispersion relation.

We show in Figure 2 the dependence of acoustoelectric current density on temperature for different wave vectors. As the temperature increases, the acoustoelectric current density increases initially, reaches a maximum and falls off with increasing temperature, i.e. negative conductivity. The peak shifts with increasing $q$ towards higher temperatures for both FSWCNT and SWCNT. This suggests that higher current is obtained at lower $q$ values and at low temperatures i.e. around room temperature for FSWCNT and at temperatures below 100 $\mathrm{K}$ for SWCNT. This suggests that, the FSWCNT can be operated as an acoustoelectric device at room temperature as compared to the SWCNT. Again, increasing the value of $q$ decreases the current density for FSWCNT but the reverse occurs in SWCNT. This is because the SWCNTs are metallic and at any point in time have more intra-mini-band electrons interacting strongly through deformation or piezoelectric potential to generate higher current. Thus, higher acoustoelectric current is obtained in the SWCNT ( $\approx$ five orders of magnitude) than in the FSWCNT as shown in Figure 2.

To put our results in perspective, 3-dimensional plot is made for the acoustoelectric current density against wavenumber $(q)$ and temperature $(T)$ in Figure 3.

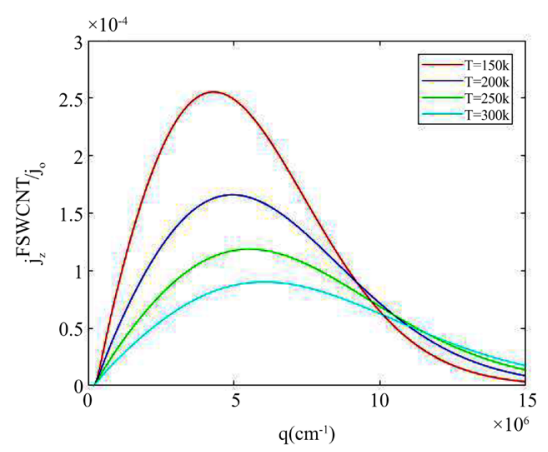

(a)

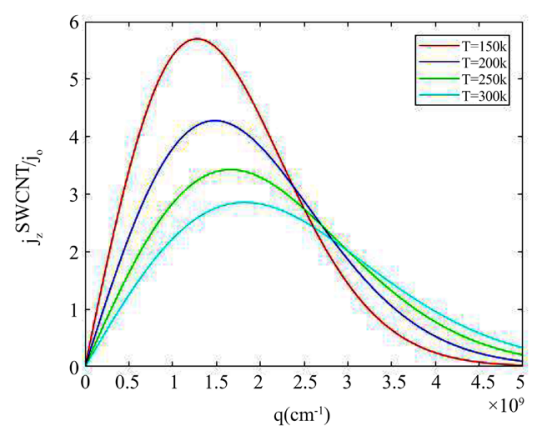

(b)

Figure 1. Dependence of AE current density on $q$ at different temperatures $T=150$ $K, T=200 K, T=250 K, T=300 K$ for (a) FSWCNT (b) SWCNT. 


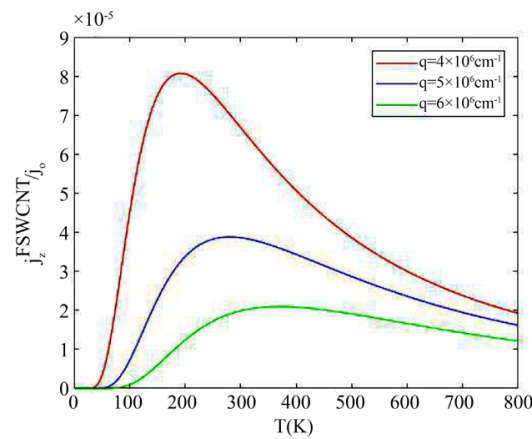

(a)

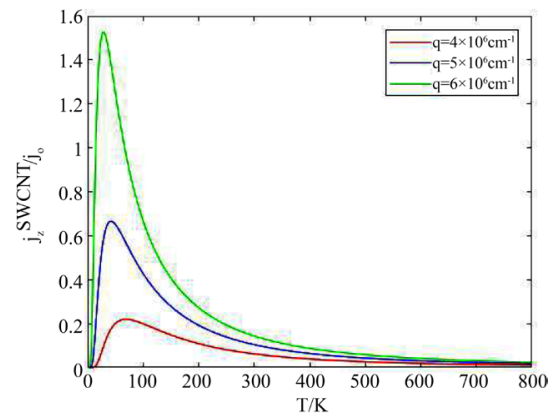

(b)

Figure 2. Dependence of AE current density on temperature $T$ for different wavevectors $q=4 \times 105, q=5 \times 105$ and $q=6 \times 105$ (a) FSWCNT (b) SWCNT.

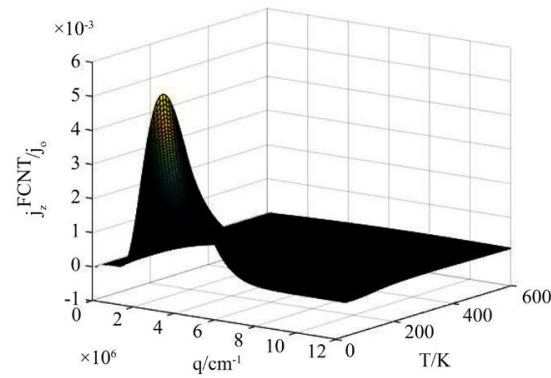

(a)

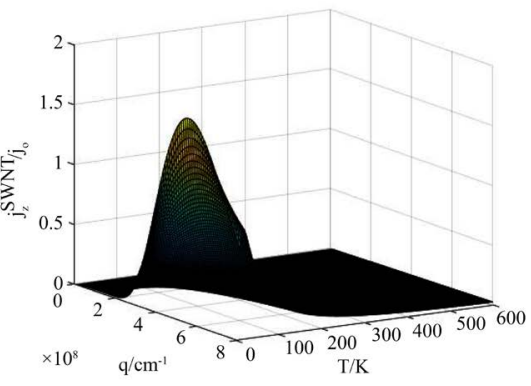

(b)

Figure 3. 3- $D$ representation of $j_{z} / j_{0}$ on $T$ and $q$ for (a) FSWCNT (b) SWCNT.

\section{Conclusion}

Acoustoelectric effect was theoretically studied in a non-degenerate semiconductor FSWCNT and undoped SWCNT using the Boltzmann's transport equation in the hypersound regime $q l \gg 1$. When $E(t)=0$, a phenomenon of negative conductivity is observed due to the strong non-parabolicity of FSWCNT and SWCNT band structure. Comparatively, the acoustoelectric current density is observed to be far less in FSWCNT than in SWCNT $\left(j_{z}^{F S W C N T} / j_{o} \ll j_{z}^{S W C N T} / j_{o}\right)$. This is because the electron-phonon interactions in SWCNT are stronger than that of FSWCNT. Thus, there are more intra-mini-band electrons interacting with the acoustic phonons to generate the acoustoelectric current in SWCNT than in FSWCNT. The study shows the potential for FSWCNT as an ultrasound current source density imaging (UCSDI) and AE hydrophone material. However, FSWCNT offers the potential for room temperature acoustoelectric device but modern techniques are needed to reduce the electrical resistance.

\section{Conflicts of Interest}

The authors declare no conflicts of interest regarding the publication of this paper.

\section{References}

[1] Galperin, Y.M. and Kagan, V.D. (1969) Aoustoelectric Effect in a Magnetic Field. 
Soviet Physics Solids State, USSR, 10, 1600.

[2] Abdelraheem, S.K., Blyth, D.P. and Balkan, N. (2001) Amplification of Ultrasonic Waves in Bulk GaN and GaAlN/GaN Heterostructures. Physica Status Solidi $(A)$, 185, 247-256. https://doi.org/10.1002/1521-396X(200106)185:2<247::AID-PSSA247>3.0.CO;2-H

[3] Hutson, A.R., McFee, I.H. and White, D.L. (1961) Ultrasonic Amplification in CdS. Physical Review Letters, 7, 273. https://doi.org/10.1103/PhysRevLett.7.237

[4] Weinreich, G. (1956) Acoustodynamic Effects in Semiconductors. Physical Review Journals Archive, 104, 321. https://doi.org/10.1103/PhysRev.104.321

[5] Mensah, S.Y. and Allotey, F.K.A. (1994) AE Effect in Semiconductor SL. Journal of Physics: Condensed Matter, 6, 6783. https://doi.org/10.1088/0953-8984/6/34/010

[6] Mensah, S.Y. and Allotey, F.K.A. (2000) Nonlinear AE Effect in Semiconductor SL's. Journal of Physics. Condensed Matter, 12, 5225. https://doi.org/10.1088/0953-8984/12/24/313

[7] Wang, Z. (2011) Biomedical Applications of Acoustoelectric Effect.

[8] Ford, C.J.B. (2017) Transporting and Manipulating Single Electrons in Surface-Acoustic-Wave Minima. Physica Status Solidi(B), 254, Article ID: 1600658. https://doi.org/10.1002/pssb.201600658

[9] Couto Jr., O.D.D., Lazić, S., Iikawa, F., Stotz, J.A.H., Jahn, U., Hey, R. and Santos, P.V. (2009) Photon Anti-Bunching in Acoustically Pumped Quantum Dots. Nature Photonics, 3, 645-648. https://doi.org/10.1038/nphoton.2009.191

[10] Hermelin, S., Takada, S., Yamamoto, M., Tarucha, S., Wieck, A.D., Saminadayar, L., Bauerle, C. and Meunier, T. (2011) Electrons Surfing on a Sound Wave as a Platform for Quantum Optics with Flying Electrons Nature, 477, 435-438. https://doi.org/10.1038/nature10416

[11] Epshtein, E.M. and Gulyaev, Y.V. (1967) AE Effect in CdS Pole Semiconductor. Soviet Physics-Solid State, 9, 288.

[12] Mori, N. and Ando, T. (1989) Electronoptical-Phonon Interaction in Single and Double Heterostructures. Physical Review B, 40, 6175. https://doi.org/10.1103/PhysRevB.40.6175

[13] Pozela, J. and Juciene, V. (2000) Electron Mobility and electron Scattering by Polar Optical Phonons in Heterostructure Quantum Wells. Semiconductors, 34, 1011-1015.

[14] Vasilopoulos, P., Charbonneau, M. and van Vlier, C.N. (1987) Linear and Nonlinear Electrical Conduction in Quasi-Two-Dimensional Quantum-Wells. Physical Review $B, 35,1334$. https://doi.org/10.1103/PhysRevB.35.1334

[15] Suzuki, A. (1992) Theory of Hot-Electron Magnetophonon Resonance in Quasitwo-Dimensional Quantum Well Structure. Physical Review B, 45, 6731. https://doi.org/10.1103/PhysRevB.45.6731

[16] Shmelev, G.M., Chaikovskii, L.A. and Bau, N.Q. (1978) High-Frequency Conductivity of Semiconductors with Superlattice. Soviet Physics Solids State, USSR, 12, 1149-1152.

[17] Bandhu, L. and Nash, G.R. (2014) Temperature Dependence of the Acoustoelectric Current in Graphene. Applied Physics Letters, 105, Article ID: 263106. https://doi.org/10.1063/1.4905222

[18] Dompreh, K.A., Mensah, N.G., Mensah, S.Y., Sam, F. and Twum, A.K. (2015) Acoustoelectric Effect in Degenerate Carbon Nanotube. ArXiv Preprint arXiv: 1504.05484.

[19] Reulet, B., Yu Kasumov, A., Kociak, M., Deblock, R., Khodos, I.I., Gorbatov, Y.B., 
Volkov, V.T., Journet, C. and Bouchiat, H. (2000) Acoustoelectric Effects in Carbon Nanotubes. Physical Review Letters, 85, 2829. https://doi.org/10.1103/PhysRevLett.85.2829

[20] Mensah, S.Y., Allotey, F.K.A., Nkrumah, G. and Mensah, N.G. (2004) High Electron Thermal Conductivity of Chiral Carbon Nanotubes. Physica E, 152, https://doi.org/10.1016/j.physe.2004.01.017

[21] Matthew, A., Abukari, S.S., Adu, K.W., Mensah, S.Y. and Mensah, N.G. (2015) Effect of Hot Electrons on the Electrical Conductivity of Carbon Nanotubes under the Influence of Applied DC Field. The European Physical Journal B, 88, 13. https://doi.org/10.1140/epjb/e2014-50493-5

[22] Sekyi-Arthur, D., Mensah, S.Y., Mensah, N.G., Dompreh, K.A. and Edziah, R. (2016) Absorption of Acoustic Phonons in Fluorinated Carbon Nanotubes with Non-Parabolic, Double Periodic Band. ArXiv Preprint arXiv:1604.05699.

[23] Sadykov, N.R., Kocherga, E.Y. and D’yachkov, P.N. (2013) Nonlinear Current in Modified Nanotubes with Exposure to Alternating and Constant Electric Fields. Russian Journal of Inorganic Chemistry, 58, 951-955. https://doi.org/10.1134/S0036023613080202

[24] Keisuke, S., Reimann, K., Woerner, M., Elsaesser, T., Hey, R. and Flytzanis, C. (2016) Strong Amplification of Coherent Acoustic Phonons by Intraminiband Currents in a Semiconductor Superlattice. Physical Review Letters, 116, Article ID: 075504. https://doi.org/10.1103/PhysRevLett.116.075504

[25] Jeon, T.-I., Son, J.-H., An, K.H., Lee, Y.H. and Lee, Y.S. (2005) Terahertz Absorption and Dispersion of Fluorine-Doped Single-Walled Carbon Nanotube. Journal of Applied Physics, 98, 34316-34316.

[26] Kryuchkov, S.V. and Mikheev, N.P. (1982) Characteristics of the Acoustoelectric Effect in Narrow-Gap Semiconductors. Soviet Physics Solids State, USSR, 16, 104.

[27] Ridley, B.K. and Watkins, T.B. (1961) Negative Resistance and High Electric Field Capture Rates in Semiconductors. Journal of Physics and Chemistry of Solids, 22, 155-158. https://doi.org/10.1016/0022-3697(61)90256-6 